\title{
Preventing numerical oscillations in the flux-split based finite difference method for compressible flows with discontinuities, II
}

\author{
Zhiwei $\mathrm{He}^{1}$, Yousheng Zhang ${ }^{1}$, Xinliang $\mathrm{Li}^{2}$ and Baolin Tian ${ }^{1, *, \dagger}$ \\ ${ }^{1}$ Laboratory of Computational Physics, Institute of Applied Physics and Computational Mathematics, \\ Beijing 100094, China \\ ${ }^{2}$ LHD, Institute of Mechanics, CAS, Beijing 100190, China
}

\begin{abstract}
SUMMARY
Problems in the characteristic-wise flux-split based finite difference method when compressible flows with contact discontinuities or material interfaces are computed were presented and analyzed. The current analysis showed the following: (i) Even with the local characteristic decomposition technique, numerical errors could be caused by point-wise flux vector splitting (FVS) methods, such as the Steger-Warming FVS or the van Leer FVS. Therefore, the Lax-Friedrichs type FVS method is required. (ii) If the isobars of a material are vertical lines, the combination of using the local characteristic decomposition and the global LaxFriedrichs FVS can avoid velocity and pressure oscillations of contact discontinuities in this material for weighted essentially non-oscillatory (WENO) schemes. (iii) For problems with material interfaces, the quasiconservative approach can be realized using characteristic-wise flux-split based finite difference WENO schemes if nonlinear WENO schemes in genuinely nonlinear characteristic fields can be guaranteed to be the same and the decomposition equation representing material interfaces is discretized properly. Copyright (C) 2015 John Wiley \& Sons, Ltd.
\end{abstract}

Received 23 March 2015; Revised 16 July 2015; Accepted 17 July 2015

KEY WORDS: contact discontinuity; material interface; finite difference method; flux vector splitting; local characteristic decomposition; equation of state; WENO

\section{INTRODUCTION}

It is well known that numerical solutions of a conservative scheme show spurious oscillation in pressure and velocity in compressible flows with contact discontinuities or material interfaces [1-5]. For single-material contact discontinuities, numerical oscillations may be caused by nonlinear equation of state (EOS) [1] and component-wise reconstruction of conservative variables [3]. Numerical oscillations caused by the component-wise reconstruction of conservative variables can be avoided by reconstructing primitive or characteristic variables [3]. However, to avoid numerical oscillations caused by nonlinear EOS, the conservative Godunov-type method might have to be abandoned [1, 2]. Recently, Lee et al. [2] developed an adaptive framework by switching between a primitive and a conservative numerical scheme for the Euler equations with highly nonlinear EOSs, yielding good results. In problems with material interfaces, pressure and velocity oscillations are also introduced when conservative methods are used $[4,5]$. A fully conservative Godunov-type scheme cannot maintain pressure equilibrium and will develop a pressure oscillation across material interfaces [5]. In order to overcome this difficulty, several fully nonconservative [6] and non-strictly conservative [7-11] approaches have been proposed. Among these attempts, the quasi-conservative approach proposed by Abgrall [9] appears promising and has been extended to problems with

\footnotetext{
*Correspondence to: Baolin Tian, Institute of Applied Physics and Computational Mathematics, Beijing 100094, China.

${ }^{\dagger}$ E-mail: tian_baolin@iapcm.ac.cn 
complicated EOSs [12-14]. This approach has been realized with low-order finite volume methods (FVM), such as first-order and second-order variable reconstruction with various Riemann solvers [15]. Recently, this approach has been extended to high-order weighted essentially non-oscillatory (WENO) reconstruction with the Harten-Lax-van Leer-Contact solver [16] by Johnsen et al. [17, 18]. They suggested that only the reconstruction of primitive variables can eliminate the spurious oscillations because the specific heat ratio is not constant [17].

On the other hand, finite difference method (FDM), particularly the flux-split based FDM, is widely used in simulations of multi-scale problems [19]. However, the types of computational difficulties associated with the use of flux-split based FDM, particularly the frequently used nonlinear FD WENO schemes, in solving problems of compressible flows with contact discontinuities and/or material interfaces are not known. As argued by Nonomura et al. [20], the technique proposed by Johnsen and Colonius [17] cannot be applied to flux-split based FD WENO schemes because these schemes do not include primitive variable reconstruction.

In [21], we revealed that the two steps in the flux-split based FDM result in numerical oscillations [21]. In the first step, flux vector splitting (FVS) methods, such as the Steger-Warming FVS [22] and van Leer FVS [23], are frequently used to split the flux vector into positive and negative flux vectors. However, these methods do not prevent velocity and pressure oscillations [5, 24]. Such FVS methods are called point-wise FVS methods. In contrast, the Lax-Friedrichs type FVS method proposed by Shu $[25,26]$ is found to have the ability to prevent this difficulty, and the global Lax-Friedrichs FVS [26] was identified as the most simple and efficient FVS method [21]. In the second step, FD schemes such as WENO schemes are implemented to obtain the numerical flux. The popular method is to use the schemes in each equation directly; this may be described as being implemented in a component-wise fashion [26]. However, nonlinear component-wise WENO discretization can also cause numerical oscillations, and such numerical oscillations should be avoided by special treatments of implementing WENO schemes consistently [21].

In fact, FD schemes can also be implemented in a characteristic-wise fashion [26] to obtain the numerical flux. Through numerical simulations, Qiu and Shu [27] showed that if the characteristicwise flux-split based FDM was used, that is, the local characteristic decomposition technique was adopted in the second step of the flux-split based FDM, essentially nonoscillatory solutions of WENO schemes could be obtained. Therefore, it is important to determine whether the characteristic-wise flux-split based FDM can prevent oscillations of velocity and pressure in compressible flows with contact discontinuities or material interfaces. This is the topic of the present work. The following conclusions were deduced:

- Even with the local characteristic decomposition technique, numerical errors could be caused by point-wise FVS methods, such as the Steger-Warming FVS or the van Leer FVS. In contrast, this difficulty can be prevented by using the Lax-Friedrichs-type FVS method proposed by Shu [25, 26], and the global Lax-Friedrichs FVS [26] was identified as the most simple and efficient FVS method.

- It can be theoretically proven that if the isobars of a material are vertical lines, the combination of using the local characteristic decomposition and the global Lax-Friedrichs FVS can prevent velocity and pressure oscillations of contact discontinuities in this material for WENO schemes.

- For problems with material interfaces, the quasi-conservative approach proposed by Abgrall [9] can be realized using characteristic-wise flux-split based FD WENO schemes if nonlinear WENO schemes in genuinely nonlinear characteristic fields are guaranteed to be the same and the decomposition equation representing material interfaces is discretized properly.

The paper is organized as follows. Section 2 focuses on the governing equations and numerical framework. Section 3 describes analyses of numerical errors. Numerical tests are described in Section 4. The conclusions are given in Section 5. 


\section{GOVERNING EQUATIONS AND NUMERICAL METHODOLOGY}

\subsection{Governing equations}

The following one-dimensional Euler equation was considered:

$$
\mathbf{u}_{t}+[\mathbf{f}(\mathbf{u})]_{x}=0
$$

where $\mathbf{u}$ and $\mathbf{f}$ are given in the following form:

$$
\mathbf{u}=\left(\begin{array}{c}
\rho \\
\rho u \\
\rho E
\end{array}\right), \mathbf{f}=\left(\begin{array}{c}
\rho u \\
\rho u^{2}+p \\
(\rho E+p) u
\end{array}\right)
$$

Here, $\rho$ is the density, $u$ is the velocity, $E=\rho e+\frac{u^{2}}{2}$ is the total energy, $e$ is the specific internal energy, and $p$ is the pressure. In this study, the Mie-Grüneisen type EOS $p=\Gamma(\rho) \rho e+g(\rho)$ [2] was used, where $\Gamma(\rho)$ is the Grüneisen coefficient defined as $\Gamma(\rho)=\left.\frac{1}{\rho} \frac{\partial p}{\partial e}\right|_{\rho}$ and the function $g(\rho)$ is defined in [2]. Accordingly, the sound speed $c^{2}=\left.\frac{\partial p}{\partial \rho}\right|_{e}+\left.\frac{p}{\rho^{2}} \frac{\partial p}{\partial e}\right|_{\rho}$.

\subsection{Numerical methodology}

After the spatial domain was discretized into an $N$-point grid with uniform grid spacing $\Delta x$, the mass, momentum, and energy conservation equations at point $x_{i}$ could be updated by one time step $(\Delta t)$ or substep:

$$
\mathbf{u}_{i}^{n+1}=\mathbf{u}_{i}^{n}-\frac{\Delta t}{\Delta x} \mathbf{F}_{i},
$$

where $\mathbf{F}_{i}=\left(F_{i}^{\rho}, F_{i}^{\rho u}, F_{i}^{\rho E}\right)^{T}$ is defined as $\mathbf{F}_{i} \equiv \widehat{\mathbf{f}}_{i+1 / 2}-\widehat{\mathbf{f}}_{i-1 / 2}$. Here, $\widehat{\mathbf{f}}_{i \pm 1 / 2}$ is the numerical flux at $(i \pm 1 / 2)$.

From Equation (2), the velocity and internal energy per unit volume at time $t^{n+1}$ are given by

$$
\begin{gathered}
u_{i}^{n+1}=\frac{(\rho u)_{i}^{n+1}}{\rho_{i}^{n+1}}=\frac{(\rho u)_{i}^{n}-F_{i}^{\rho u} \Delta t /(\Delta x)}{\rho_{i}^{n}-F_{i}^{\rho} \Delta t /(\Delta x)}, \\
(\rho e)_{i}^{n+1}=(\rho e)_{i}^{n}-\frac{\Delta t}{\Delta t} F_{i}^{\rho E}+\frac{\left[(\rho u)_{i}^{n}\right]^{2}}{2 \rho_{i}^{n}}-\frac{\left[(\rho u)_{i}^{n}-F_{i}^{\rho u} \Delta t /(\Delta x)\right]^{2}}{2\left[\rho_{i}^{n}-F_{i}^{\rho} \Delta t /(\Delta x)\right]} .
\end{gathered}
$$

The flux-split based FDM usually involves two steps. The first step is usually to split the flux vector into positive flux $\mathbf{f}^{+}$and negative flux vectors $\mathbf{f}^{-}$as $\mathbf{f}=\mathbf{f}^{+}+\mathbf{f}^{-}$, where the two Jacobians $\frac{\partial \mathbf{f}^{+}}{\partial \mathbf{u}}, \frac{\partial \mathbf{f}^{-}}{\partial \mathbf{u}}$ are still diagonalizable and have only non-negative/non-positive eigenvalues [26]. In [21], as the ideal-gas EOS was considered, in order to make a clear comparison between different FVS methods for the simplest example of a problem: a single-material stationary contact discontinuity discretized by the first-order upwind difference scheme, we presented a unified form of the StegerWarming FVS and Lax-Friedrichs type FVS as

$$
\mathbf{f}^{ \pm}=R \frac{\Lambda \pm \Lambda^{*}}{2} L \mathbf{u}
$$

where $R$ and $L$ are matrices formed by the right and left eigenvectors, respectively, of the Jacobian $\frac{\partial \mathbf{f}}{\partial \mathbf{u}} . \Lambda$ is a diagonal matrix formed by the corresponding eigenvalues $\lambda_{k}(k=1, \cdots, 3)$ of $\frac{\partial \mathbf{f}}{\partial \mathbf{u}}$, which are ordered as $\lambda_{1}=u-c, \lambda_{2}=u, \lambda_{3}=u+c$, where $c$ is the local sound speed. The diagonal matrix $\Lambda^{*}$ formed by $\lambda_{k}^{*}(k=1, \cdots, 3)$ differs for different FVS methods as follows: (i) in the Steger-Warming FVS, $\Lambda^{*}=|\Lambda|$; and (ii) in the global Lax-Friedrichs FVS, $\Lambda^{*}=\alpha I$, where $I$ is the identity matrix and $\alpha=\max _{1 \leqslant j \leqslant 3}\left|\lambda_{j}\right|$ of the whole computation domain [26]. In fact, the global Lax-Friedrichs FVS is directly written as 


$$
\mathbf{f}^{ \pm}=\frac{\mathbf{f} \pm \alpha \mathbf{u}}{2}
$$

This method does not require the flux function to be a homogeneous function of degree one so that the flux function can be expressed as a product of the Jacobian matrix and conservative variables. Therefore, this method is suitable for materials described by complicated EOS.

The next step is to obtain the numerical flux via various conservative FD schemes. The characteristic-wise fashion [26, 27] involves the following steps.

At each fixed $x_{i+1 / 2}$, the average state $\mathbf{u}_{i+1 / 2}$ is computed by the simple mean or the Roe mean. The corresponding eigenvalue, left eigenvector, and right eigenvector are denoted by $\lambda_{k, i+1 / 2}$, $\mathbf{l}_{k, i+1 / 2}^{T}$, and $\mathbf{r}_{k, i+1 / 2}$, where $k=1, \cdots, 3$.

The local characteristic decomposition of the flux function at $x_{m}$ are computed using $\mathbf{q}_{m}^{+}=$ $L_{i+1 / 2} \mathbf{f}_{m}^{+}$and $\mathbf{q}_{m}^{-}=L_{i+1 / 2} \mathbf{f}_{m}^{+}$, where the matrix $L_{i+1 / 2}$ is formed by the row vectors $\mathbf{l}_{k, i+1 / 2}^{T}$ and $m=i-l, \cdots, i+r$ labels the points from the leftmost to the rightmost in the stencil of the conservative scheme. Using the nonlinear scheme to obtain the characteristic numerical flux in each characteristic field, we obtain $\widehat{q}_{k, i+1 / 2}^{+}$and $\widehat{q}_{k, i+1 / 2}^{-}$. Then, we obtain $\widehat{\mathbf{q}}_{i+1 / 2}^{+}$and $\widehat{\mathbf{q}}_{i+1 / 2}^{-}$.

Finally, transforming back into physical space, we obtain $\widehat{\mathbf{f}}_{i+1 / 2}=\widehat{\mathbf{f}}_{i+1 / 2}^{+}+\widehat{\mathbf{f}}_{i+1 / 2}^{-}=$ $R_{i+1 / 2}\left(\widehat{\mathbf{q}}_{i+1 / 2}^{+}+\widehat{\mathbf{q}}_{i+1 / 2}^{-}\right)$, where the matrix $R_{i+1 / 2}$ is formed by the column vectors $\mathbf{r}_{k, i+1 / 2}$.

\section{NUMERICAL ERROR ANALYSIS}

Noticing that the velocity $u$ and pressure $p$ were constant at time $t^{n}$, we denoted them as $u_{0}$ and $p_{0}$. Using the specific expression of $R_{i+1 / 2}$,

$$
R_{i+1 / 2}=\left(\begin{array}{ccc}
1 & 1 & 1 \\
u_{0}-c_{i+1 / 2} & u_{0} & u_{0}+c_{i+1 / 2} \\
s_{i+1 / 2}+\frac{u_{0}^{2}}{2}-u_{0} c_{i+1 / 2} & \frac{u_{0}^{2}}{2}+b_{i+1 / 2} & s_{i+1 / 2}+\frac{u_{0}^{2}}{2}+u_{0} c_{i+1 / 2}
\end{array}\right)
$$

where the $s_{i+1 / 2}\left(\equiv e_{i+1 / 2}+\frac{p_{0}}{\rho_{i+1 / 2}}\right)$, sound speed $c_{i+1 / 2}$, Grüneisen coefficient $\Gamma_{i+1 / 2}$, and $b_{i+1 / 2}\left(\equiv s_{i+1 / 2}-\frac{c_{i+1 / 2}^{2}}{\Gamma_{i+1 / 2}}\right)$ were calculated in terms of $\mathbf{u}_{i+1 / 2}$, we have,

$$
\widehat{\mathbf{f}}_{i+1 / 2}^{ \pm}=\left(\begin{array}{c}
\sum_{k=1}^{3} \widehat{q}_{k, i+1 / 2}^{ \pm} \\
u_{0}\left(\sum_{k=1}^{3} \widehat{q}_{k, i+1 / 2}^{ \pm}\right)+c_{i+1 / 2} Q_{M, i+1 / 2}^{ \pm} \\
\frac{u_{0}^{2}}{2}\left(\sum_{k=1}^{3} \widehat{q}_{k, i+1 / 2}^{ \pm}\right)+u_{0} c_{i+1 / 2} Q_{M, i+1 / 2}^{ \pm}+s_{i+1 / 2} Q_{P, i+1 / 2}^{ \pm}+b_{i+1 / 2} \widehat{q}_{2, i+1 / 2}^{ \pm}
\end{array}\right),
$$

where

$$
\begin{aligned}
& Q_{M, i+1 / 2}^{ \pm}=\widehat{q}_{3, i+1 / 2}^{ \pm}-\widehat{q}_{1, i+1 / 2}^{ \pm}, \\
& Q_{P, i+1 / 2}^{ \pm}=\widehat{q}_{3, i+1 / 2}^{ \pm}+\widehat{q}_{1, i+1 / 2}^{ \pm}
\end{aligned}
$$

\subsection{Necessity of Lax-Friedrichs type flux vector splitting}

In [21], we reported that the general condition for Equation (5), coupled with the first-order upwind scheme to avoid velocity a nd pressure oscillations for a stationary contact discontinuity, is that $\left(\lambda_{1}^{*}\right)_{m}=\left(\lambda_{3}^{*}\right)_{m}=\left(\lambda_{0}^{*}\right)_{m}$ for a fixed point $x_{m}$ and $\left(\lambda_{0}^{*}\right)_{m}$ should be kept constant for arbitrary values of $m$, where $m=i-l, \cdots, i+r$ labels the points from the leftmost point to the rightmost point in the stencil of the conservative scheme. In order to avoid introducing numerical oscillations by FVS for contact discontinuities, the relation $\left(\left(\lambda_{1}^{*}\right)_{m}=\left(\lambda_{2}^{*}\right)_{m}=\left(\lambda_{3}^{*}\right)_{m}=\right.$ const $)$ should hold for arbitrary point in the stencil. Therefore, although the equations are decoupled when the local characteristic decomposition technique is used, the Steger-Warming FVS will still introduce numerical 
oscillations because $\left(\lambda_{k}^{*}\right)_{i}(k=1, \cdots, 3 ; i=1, \cdots, N)$ is different for different grid points. In contrast, the global Lax-Friedrichs FVS was found to be the most simple and efficient method to satisfy these constraints in which $\left(\lambda_{k}^{*}\right)_{m}$ is set to a constant $\alpha$ for the entire computation field and is used as the foundation of analyses hereafter.

\section{Remark 1}

When the local characteristic decomposition technique is adopted, the local Lax-Friedrichs flux splitting method [25] can also be used in each characteristic field. Following the notations used in this paper, the local Lax-Friedrichs flux splitting can be written as

$$
q_{k, m}^{ \pm}=\frac{1}{2} \mathbf{l}_{k, i+1 / 2}^{T}\left(\mathbf{f}_{m} \pm \alpha_{k, i+1 / 2} \mathbf{u}_{m}\right),
$$

where $\alpha_{k, i+1 / 2}\left(\equiv \max \left(\left|\left(\lambda_{k}\right)_{i}\right|,\left|\left(\lambda_{k}\right)_{i+1}\right|\right)\right)$ at $i+1 / 2$ in the $k$ th characteristic field $[25,28]$. The following conclusions are still valid for the local Lax-Friedrichs flux splitting method. However, this method cannot be used in a component-wise fashion.

\subsection{One-dimensional contact discontinuity problem}

In [21], we reported that even with the ideal-gas EOS, numerical oscillations can be caused by component-wise nonlinear difference discretization among equations. In this section, we present the following theoretical derivation:

- For a material whose isobars are vertical lines in the $(\rho e, \rho)$ plane, that is, $\Gamma_{i+1 / 2}(\rho)=\Gamma$, $g(\rho)=g, s_{i+1 / 2}=\frac{c_{i+1 / 2}^{2}}{\Gamma_{i+1 / 2}}$ and $b_{i+1 / 2}=0$, by combining the local characteristic decomposition and the global Lax-Friedrichs FVS, velocity and pressure oscillations of contact discontinuities can be avoided for WENO schemes.

When the global Lax-Friedrichs FVS is used, the local positive/negative characteristic fluxes can be written as

$$
\mathbf{q}_{m}^{ \pm}=\frac{\Gamma_{i+1 / 2}}{2 c_{i+1 / 2}^{2}}\left(\begin{array}{lcc}
\frac{u_{0}^{2}}{2}+\frac{u_{0} c_{i+1 / 2}}{\Gamma_{i+1 / 2}} & -u_{0}-\frac{c_{i+1 / 2}}{\Gamma_{i+1 / 2}} & 1 \\
\frac{2 c_{i+1 / 2}^{2}}{\Gamma_{i+1 / 2}}-u_{0}^{2} & 2 u_{0} & -2 \\
\frac{u_{0}^{2}}{2}-\frac{u_{0} c_{i+1 / 2}}{\Gamma_{i+1 / 2}} & -u_{0}+\frac{c_{i+1 / 2}}{\Gamma_{i+1 / 2}} & 1
\end{array}\right)\left(\begin{array}{c}
\frac{(\rho u)_{m} \pm \alpha \rho_{m}}{2} \\
\frac{\left(\rho u^{2}+p\right)_{m} \pm \alpha(\rho u)_{m}}{2} \\
\frac{(\rho E u+p u)_{m} \pm \alpha(\rho E)_{m}}{2}
\end{array}\right) .
$$

For a contact discontinuity, the velocity and pressure are constant and the local positive/negative characteristic fluxes can be simplified into

$$
\mathbf{q}_{m}^{ \pm}=\left(\begin{array}{c}
-\frac{1}{2 c_{i+1 / 2}} \frac{p_{0}}{2}+\frac{\Gamma_{i+1 / 2}}{2 c_{i+1 / 2}^{2}} \frac{u_{0} \pm \alpha}{2}\left(\frac{p_{0}-g}{\Gamma}\right)_{m} \\
\frac{u_{0} \pm \alpha}{2} \frac{2 c_{i+1 / 2}^{2}-\Gamma_{i+1 / 2} u_{0}}{2 c_{i+1 / 2}^{2}} \rho_{m}-\frac{\Gamma_{i+1 / 2}}{c_{i+1 / 2}^{2}} \frac{u_{0} \pm \alpha}{2}\left(\frac{p_{0}-g}{\Gamma}\right)_{m} \\
\frac{1}{2 c_{i+1 / 2}} \frac{p_{0}}{2}+\frac{\Gamma_{i+1 / 2}}{2 c_{i+1 / 2}^{2}} \frac{u_{0} \pm \alpha}{2}\left(\frac{p_{0}-g}{\Gamma}\right)_{m}
\end{array}\right)
$$

By denoting the discretization operator of WENO schemes used in the $k$ th characteristic field as $D_{k, i+1 / 2}^{ \pm}$, the characteristic numerical fluxes $\hat{q}_{1, i+1 / 2}^{ \pm}$and $\hat{q}_{3, i+1 / 2}^{ \pm}$in Equation (8) can be written as

$$
\begin{aligned}
& \widehat{q}_{1, i+1 / 2}^{ \pm}=D_{1, i+1 / 2}^{ \pm}\left(-\frac{1}{2 c_{i+1 / 2}} \frac{p_{0}}{2}\right)+D_{1, i+1 / 2}^{ \pm}\left(\frac{\Gamma_{i+1 / 2}}{2 c_{i+1 / 2}^{2}} \frac{u_{0} \pm \alpha}{2}\left(\frac{p_{0}-g}{\Gamma}\right)_{m}\right), \\
& \widehat{q}_{3, i+1 / 2}^{ \pm}=D_{3, i+1 / 2}^{ \pm}\left(\frac{1}{2 c_{i+1 / 2}} \frac{p_{0}}{2}\right)+D_{3, i+1 / 2}^{ \pm}\left(\frac{\Gamma_{i+1 / 2}}{2 c_{i+1 / 2}^{2}} \frac{u_{0} \pm \alpha}{2}\left(\frac{p_{0}-g}{\Gamma}\right)_{m}\right) .
\end{aligned}
$$


Then we have

$$
\begin{gathered}
Q_{P, i+1 / 2}^{ \pm}=D_{3, i+1 / 2}^{ \pm}\left(\psi_{i+1 / 2}^{ \pm}\left(\frac{p_{0}-g}{\Gamma}\right)_{m}\right)+D_{1, i+1 / 2}^{ \pm}\left(\psi_{i+1 / 2}^{ \pm}\left(\frac{p_{0}-g}{\Gamma}\right)_{m}\right), \\
Q_{M, i+1 / 2}^{ \pm}=\frac{p_{0}}{2 c_{i+1 / 2}}+D_{3, i+1 / 2}^{ \pm}\left(\psi_{i+1 / 2}^{ \pm}\left(\frac{p_{0}-g}{\Gamma}\right)_{m}\right)-D_{1, i+1 / 2}^{ \pm}\left(\psi_{i+1 / 2}^{ \pm}\left(\frac{p_{0}-g}{\Gamma}\right)_{m}\right),
\end{gathered}
$$

where $\psi_{i+1 / 2}^{ \pm}=\frac{\Gamma_{i+1 / 2}}{2 c_{i+1 / 2}^{2}} \frac{u_{0} \pm \alpha}{2}$.

Finally, for a single-material problem ( $\Gamma=$ const, $g=$ const), Equations (16) and (17) can be simplified further into

$$
\begin{gathered}
Q_{P, i+1 / 2}^{ \pm}=\frac{u_{0} \pm \alpha}{2} \frac{p_{0}-g}{c_{i+1 / 2}^{2}}, \\
Q_{M, i+1 / 2}^{ \pm}=\frac{p_{0}}{2 c_{i+1 / 2}} .
\end{gathered}
$$

Using Equations (19) and (8) and the definition of $\mathbf{F}_{i}$, we can obtain $F_{i}^{\rho u}=u_{0} F_{i}^{\rho}$. Substituting this relation to Equation (3), we can obtain $u_{i}^{n+1}=u_{i}^{n}=u_{0}$. Under this condition without velocity oscillation, we can similarly have $F_{i}^{\rho E}=\frac{u_{0}^{2}}{2} F_{i}^{\rho}$ by using Equations (18), (19), and (8) and the definition of $\mathbf{F}_{i}$. Substituting this relation to Equation (4), we have $\left(\frac{p-g}{\Gamma}\right)_{i}^{n+1}=\left(\frac{p-g}{\Gamma}\right)_{i}^{n}$, that is, $p_{i}^{n+1}=p_{i}^{n}=p_{0}$. Thus, the condition without pressure oscillation is obtained.

\section{Remark 2}

It should be noted that the intrinsic nonlinear mechanism of nonlinear WENO schemes may also induce numerical oscillations. It has been reported that numerical solutions are sensitive to the $\epsilon$ value, which is used to avoid division by zero in the nonlinear weights formulation [29-32]. Even for a simple linear equation such as the linear advection equation, the nonlinearity of WENO schemes can generate spurious high-mode oscillations [32]. Furthermore, these numerical oscillations will be generated regardless of the value of $\epsilon$, and the amplitude of the spurious oscillations increases with smaller $\epsilon$ values [32].

\subsection{Extension to multi-material flow}

For multi-material flows where the ratio of specific heat $\gamma$ is not constant, Abgrall [5, 9] proposed a quasi-conservative approach as follows: (i) the proper variable to represent the fluid composition is a function of the ratio of specific heat (i.e., $\phi=\frac{1}{\gamma-1}$ ); and (ii) the equation of $\phi$ should be written as

$$
\frac{\partial \phi}{\partial t}+u \frac{\partial \phi}{\partial x}=0
$$

Here, we attempted to realize Abgrall's quasi-conservative approach by using the characteristic-wise flux-split based finite difference WENO schemes.

The Grüneisen coefficient for this case is $\Gamma(\rho)=\gamma-1$ and the function $g(\rho)$ is zero. If we assume that the expressions of $D_{1, i+1 / 2}$ and $D_{3, i+1 / 2}$ are the same (denoted by $D_{0, i+1 / 2}$ ), Equations (16) and (17) can further be simplified into

$$
\begin{gathered}
Q_{P, i+1 / 2}^{ \pm}=2 D_{0, i+1 / 2}^{ \pm}\left(\psi_{i+1 / 2}^{ \pm} \frac{p_{0}}{\gamma-1}\right), \\
Q_{M, i+1 / 2}^{ \pm}=\frac{p_{0}}{2 c_{i+1 / 2}} .
\end{gathered}
$$


Using Equations (22) and (8) and the definition of $\mathbf{F}_{i}$, we can obtain $F_{i}^{\rho u}=u_{0} F_{i}^{\rho}$. Substituting this relation to Equation (3), we can obtain $u_{i}^{n+1}=u_{i}^{n}=u_{0}$. Under this velocity equilibrium, we can further obtain

$$
\begin{aligned}
F_{i}^{\rho E}=\frac{u_{0}^{2}}{2} F_{i}^{\rho} & +2 s_{i+1 / 2} D_{0, i+1 / 2}^{+}\left(\psi_{i+1 / 2}^{+} \frac{p_{0}}{\gamma-1}\right)+2 s_{i+1 / 2} D_{0, i+1 / 2}^{-}\left(\psi_{i+1 / 2}^{-} \frac{p_{0}}{\gamma-1}\right) \\
& -2 s_{i-1 / 2} D_{0, i-1 / 2}^{+}\left(\psi_{i-1 / 2}^{+} \frac{p_{0}}{\gamma-1}\right)-2 s_{i-1 / 2} D_{0, i-1 / 2}^{-}\left(\psi_{i-1 / 2}^{-} \frac{p_{0}}{\gamma-1}\right) .
\end{aligned}
$$

For WENO schemes that are combinations of low-order linear schemes [33], similar to that in [21], the constants $\psi_{i \pm 1 / 2}^{ \pm}$can be extracted from the discretization operator. Therefore, Equation (23) can be simplified into

$$
\begin{aligned}
F_{i}^{\rho E}=\frac{u_{0}^{2}}{2} F_{i}^{\rho} & +\frac{u_{0}+\alpha}{2}\left(D_{0, i+1 / 2}^{+}\left(\frac{p_{0}}{\gamma-1}\right)-D_{0, i-1 / 2}^{+}\left(\frac{p_{0}}{\gamma-1}\right)\right) \\
& +\frac{u_{0}-\alpha}{2}\left(D_{0, i+1 / 2}^{-}\left(\frac{p_{0}}{\gamma-1}\right)-D_{0, i-1 / 2}^{-}\left(\frac{p_{0}}{\gamma-1}\right)\right) .
\end{aligned}
$$

Substituting this relation to Equation (4), we can obtain

$$
\begin{aligned}
\left(\frac{p}{\gamma-1}\right)_{i}^{n+1}=\left(\frac{p}{\gamma-1}\right)_{i}^{n} & -\frac{\Delta t}{\Delta x} \frac{u_{0}+\alpha}{2}\left(D_{0, i+1 / 2}^{+}\left(\frac{p_{0}}{\gamma-1}\right)-D_{0, i-1 / 2}^{+}\left(\frac{p_{0}}{\gamma-1}\right)\right) \\
& -\frac{\Delta t}{\Delta x} \frac{u_{0}-\alpha}{2}\left(D_{0, i+1 / 2}^{-}\left(\frac{p_{0}}{\gamma-1}\right)-D_{0, i-1 / 2}^{-}\left(\frac{p_{0}}{\gamma-1}\right)\right) .
\end{aligned}
$$

If the following equation

$$
\begin{aligned}
\left(\frac{1}{\gamma-1}\right)_{i}^{n+1}=\left(\frac{1}{\gamma-1}\right)_{i}^{n} & -\frac{\Delta t}{\Delta x} \frac{u_{i}+\alpha}{2}\left(D_{0, i+1 / 2}^{+}\left(\frac{1}{\gamma-1}\right)-D_{0, i-1 / 2}^{+}\left(\frac{1}{\gamma-1}\right)\right) \\
& -\frac{\Delta t}{\Delta x} \frac{u_{i}-\alpha}{2}\left(D_{0, i+1 / 2}^{-}\left(\frac{1}{\gamma-1}\right)-D_{0, i-1 / 2}^{-}\left(\frac{1}{\gamma-1}\right)\right),
\end{aligned}
$$

is strictly held, which is simply the proper discrete form of Equation (20), the pressure equilibrium is maintained (see [21] for more information).

In general, the expressions of $D_{1, i+1 / 2}$ and $D_{3, i+1 / 2}$ are not the same. However, this constraint can be satisfied by using the common weights technique (see [21] for more information).

\section{NUMERICAL TESTS}

For the numerical tests in the following, the classical fifth-order finite difference WENO scheme (denoted as WENO5) [33] was used. The third-order total variation diminishing Runge-Kutta scheme [34] with the CFL number set to 0.01 in order to avoid any numerical errors caused by time integration was used for time integration, and initial values were fixed on the left and right boundaries of the spatial domain.

\subsection{One-dimensional isolated contact discontinuity in air}

We considered an isolated contact discontinuity, and the initial condition was

$$
(\rho, u, p)= \begin{cases}\left(2,1,1 / \gamma_{\text {air }}\right) & 0 \leqslant x \leqslant 0.1 \\ \left(0.1,1,1 / \gamma_{a i r}\right) & 0.1 \leqslant x \leqslant 2\end{cases}
$$


The computation was performed using $N=401$ grid points, with a uniform grid spacing of $\Delta x=$ 0.005 . Results at $t=0.45$ are shown in Figures 1 and 2 .

Figures 1 and 2 show the profiles of density, velocity, and pressure for the implementations of different numerical methods, and the profiles of theoretical solutions are also plotted for comparison. In Figures 1 and 2, "SW", "VL", and "GLF" denote the Steger-Warming FVS, van Leer FVS, and global Lax-Friedrichs FVS, respectively; "LCD" stands for the local characteristic decomposition technique with Roe approximation. According to our analysis, numerical oscillations of either pressure or velocity can be expected if a point-wise FVS, such as the Steger-Warming FVS or van Leer FVS, is used, and this is confirmed in Figure 2, where remarkable numerical oscillations can be observed near the location of contact discontinuity. Furthermore, the oscillations can spread into density (as shown in Figure 1), which is in contrast to that performed in the component-wise fashion [21]. The combination of using the local characteristic decomposition and global Lax-Friedrichs FVS can successfully prevent this type of numerical oscillations.

\subsection{One-dimensional isolated contact discontinuity with different equations of state}

We continued to consider an isolated contact discontinuity, but the material was described by different EOS. The first one was the stiffened EOS:

$$
p=(\gamma-1) \rho e-\gamma \Pi
$$

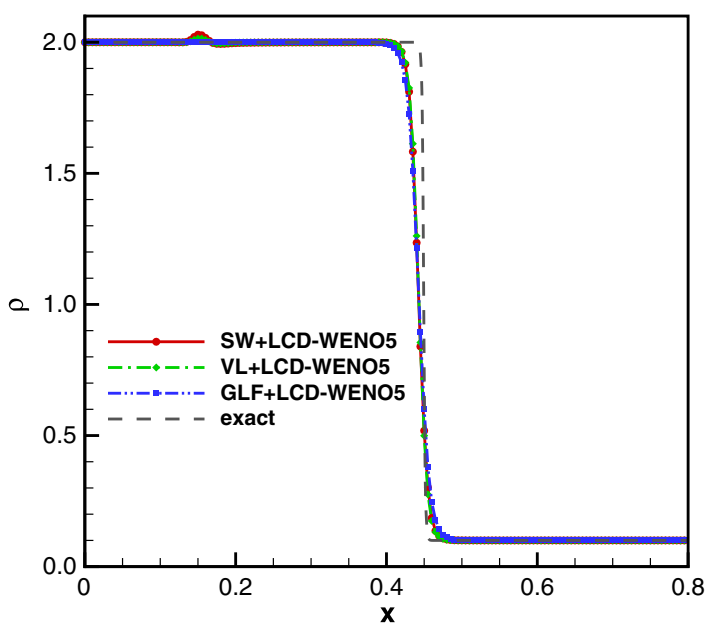

Figure 1. Density distributions at time $t=0.45$.
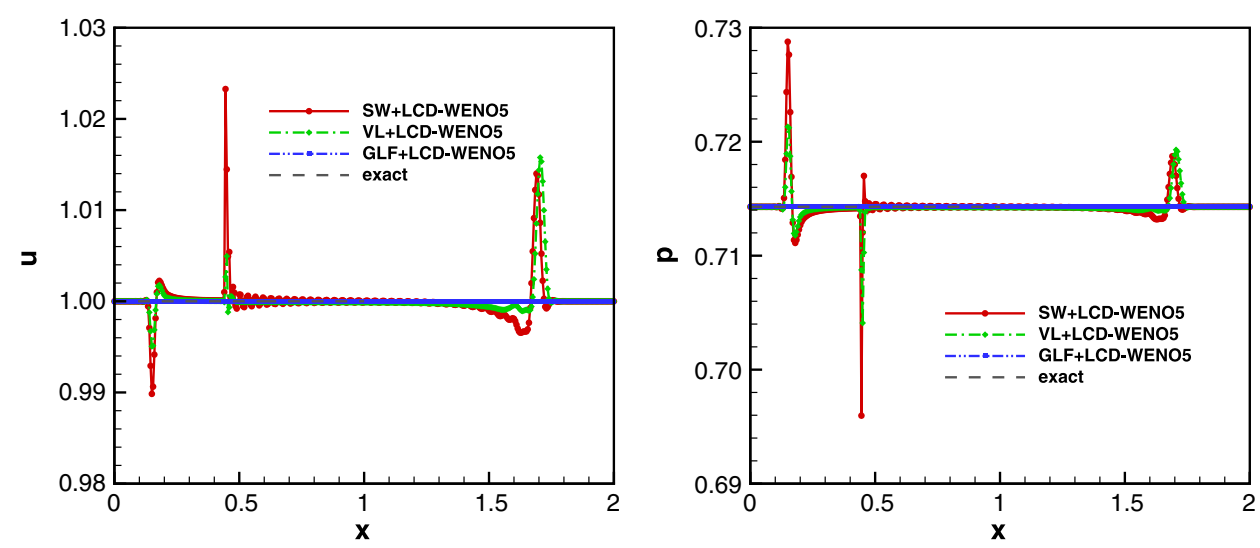

Figure 2. Velocity (left) and pressure (right) distributions at time $t=0.45$. 
where $\gamma$ is the usual ratio of specific heats $(\gamma>1)$ and $\Pi$ is a prescribed pressure-like constant with the parameter values taken in turn to be $\gamma=4.4, \Pi=6 \times 10^{8} \mathrm{~Pa}[13]$ in this paper. The second was the van der Waals EOS:

$$
p=\frac{\gamma-1}{1-\chi \rho}\left(\rho e+\zeta \rho^{2}\right)-\zeta \rho^{2},
$$

where $\gamma$ is the ratio of specific heats and the quantities $\zeta, \chi$ are the van der Waals gas constants for molecular cohesive forces and the finite size of molecules [13]. In this study, $\gamma=1.4, \zeta=5 \mathrm{~Pa}$ $\mathrm{m}^{6} / \mathrm{kg}^{2}$, and $\chi=10^{-3} \mathrm{~m}^{3} / \mathrm{kg}$ were used for the van der Waals gas [13]. The initial condition was

$$
(\rho, u, p)= \begin{cases}\left(\rho_{0}, 100 \mathrm{~m} / \mathrm{s}, 10^{5} \mathrm{~Pa}\right) & 0 \mathrm{~m} \leqslant y \leqslant 5 \mathrm{~m} \\ \left(0.1 \rho_{0}, 100 \mathrm{~m} / \mathrm{s}, 10^{5} \mathrm{~Pa}\right) & 5 \mathrm{~m} \leqslant y \leqslant 10 \mathrm{~m} .\end{cases}
$$

For the stiffened liquid, the reference density was set to $\rho_{0}=1000 \mathrm{~kg} / \mathrm{m}^{3}$, and for the van der Waals gas, the reference density was set to $\rho_{0}=50 \mathrm{~kg} / \mathrm{m}^{3}$. The Roe approximation was used for the local characteristic decomposition, and the global Lax-Friedrichs FVS was used for the numerical fluxes. This test was performed on a uniform mesh of $N=401$ grid points. Results at $t=25 \mathrm{~ms}$ are shown in Figures 3 and 4.

Figures 3 and 4 show the profiles of scaled density, velocity, and pressure of different materials. According to our analysis, if the isobars are vertical lines in the $(\rho e, \rho)$ plane, the combination of

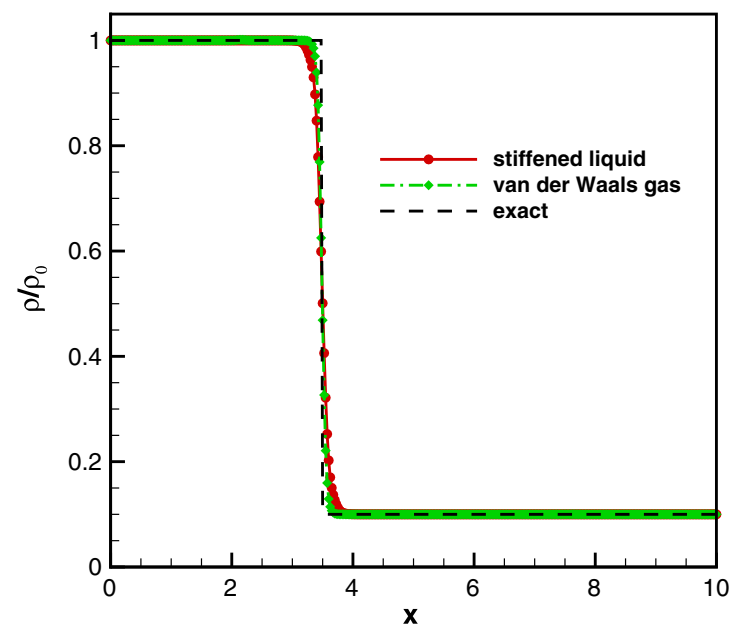

Figure 3. Scaled density distributions at time $t=25 \mathrm{~ms}$.
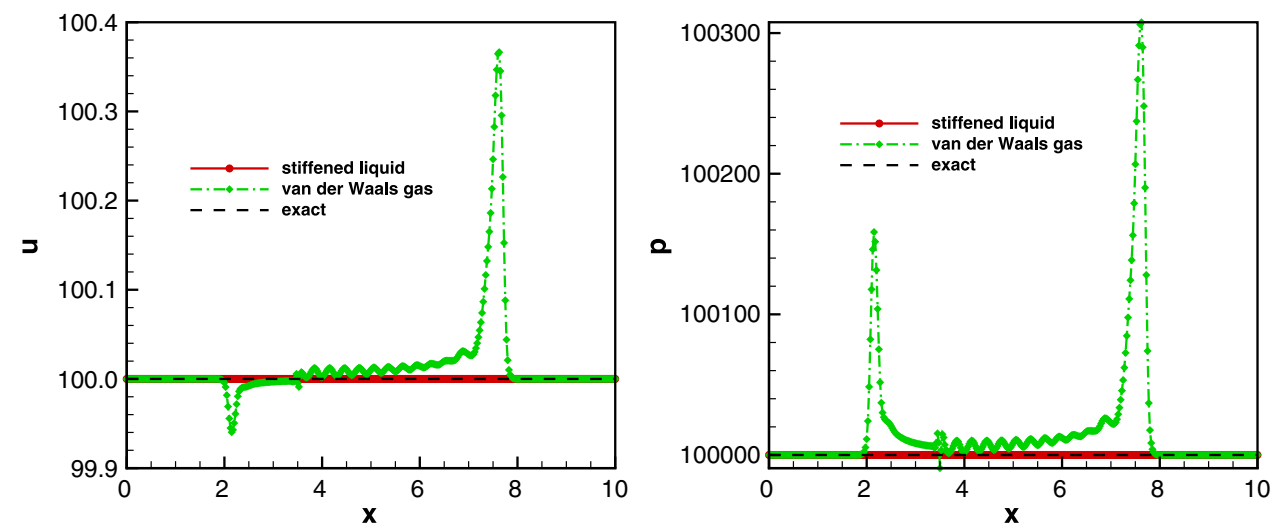

Figure 4. Velocity (left) and pressure (right) distributions at time $t=25 \mathrm{~ms}$. 
using the local characteristic decomposition and global Lax-Friedrichs FVS can prevent velocity and pressure oscillations in contact discontinuities. This claim was confirmed by the results of the stiffened liquid. In contrast, for the van der Waals gas, numerical oscillations of velocity and pressure appeared.

\section{CONCLUSION}

Two steps are involved in flux-split based FDMs: split the flux vector into the positive/negative flux and use an FD scheme to construct the numerical flux. The current analysis showed the following: (i) Even with the local characteristic decomposition technique, numerical errors could be caused by the point-wise FVS method. In contrast, the global Lax-Friedrichs FVS was found to have the ability to avoid such numerical errors; and (ii) If the isobars of a material are vertical lines, the combination of using the local characteristic decomposition and the global Lax-Friedrichs FVS can prevent velocity and pressure oscillations in contact discontinuities in this material for WENO schemes.

For problems with material interfaces, the quasi-conservative approach proposed by Abgrall [9] can be realized by the characteristic-wise flux-split based FD WENO schemes if nonlinear WENO schemes in genuinely nonlinear characteristic fields are guaranteed to be the same and the decomposition equation representing material interfaces is discretized properly.

\section{ACKNOWLEDGEMENTS}

This work was supported by the CAEP under Grant 2012A020210, NSFC under Grants 11171037, 91130021, 11472059, 11372330, 11472278, and Key Lab Foundation under Grant 9140C690104150C69.

\section{REFERENCES}

1. Clerc S. Accurate computation of contact discontinuities in flows with general equations of state. Computer Methods in Applied Mechanics and Engineering 1999; 178:245-255.

2. Lee BJ, Toro EF, Castro CE, Nikiforakis N. Adaptive Osher-type scheme for the Euler equations with highly nonlinear equations of state. Journal of Computational Physics 2013; 246:165-183.

3. Johnsen E. On the treatment of contact discontinuities using WENO schemes. Journal of Computational Physics 2011; 230:8665-8668.

4. Toro EF. Anomalies of conservative methods: analysis, numerical evidence and possible cures. Computational Fluid Dynamics Journal 2002; 11:128-143.

5. Abgrall R, Karni S. Computations of compressible multifluids. Journal of Computational Physics 2001; 169: 594-623.

6. Karni S. Multi-component flow calculations by a consistent primitive algorithm. Journal of Computational Physics 1994; 112:31-43.

7. Toro EF. Defects of conservative approaches and adaptive primitive-conservative schemes for computing solutions to hyperbolic conservation laws. Technical Report MMU 9401, Manchester Metropolitan University, UK, 1994.

8. Karni S. Hybrid multifluid algorithms. SIAM Journal on Scientific Computing 1996; 17:1019-1039.

9. Abgrall R. How to prevent pressure oscillations in multicomponent flow calculations: a quasi conservative approach. Journal of Computational Physics 1996; 125:150-160.

10. Jenny P, Müller B, Thomann H. Correction of conservative Euler solvers for gas mixtures. Journal of Computational Physics 1997; 132:91-107.

11. Fedkiw RP, Aslam T, Merriman B, Osher S. A non-oscillatory Eulerian approach to interfaces in multimaterial flows (the ghost fluid method). Journal of Computational Physics 1999; 152:457-492.

12. Shyue KM. An efficient shock-capturing algorithm for compressible multicomponent problems. Journal of Computational Physics 1998; 142:208-242.

13. Shyue KM. A fluid-mixture type algorithm for compressible multicomponent flow with van der Waals equation of state. Journal of Computational Physics 1999; 156:43-88.

14. Shyue KM. A fluid-mixture type algorithm for compressible multicomponent flow with Mie-Grüneisen equation of state. Journal of Computational Physics 2001; 171:678-707.

15. Saurel R, Abgrall R. A simple method for compressible multifluid flows. SIAM Journal on Scientific Computing 1999; 21:1115-1145.

16. Toro E, Spruce M, Speares W. Restoration of the contact surface in the HLL-Riemann solver. Shock Waves 1994; 4:25-34.

17. Johnsen E, Colonius T. Implementation of WENO schemes in compressible multicomponent flow problems. Journal of Computational Physics 2006; 219:715-732. 
18. Johnsen E, Ham F. Preventing numerical errors generated by interface-capturing schemes in compressible multimaterial flows. Journal of Computational Physics 2012; 231:5705-5717.

19. Pirozzoli S. Numerical methods for high-speed flows. Annual Review of Fluid Mechanics 2011; 43:163-194.

20. Nonomura T, Morizawa S, Terashima H, Obayashi S, Fujii K. Numerical (error) issues on compressible multicomponent flows using a high-order differencing scheme: weighted compact nonlinear scheme. Journal of Computational Physics 2012; 231:3181-3210.

21. He ZW, Zhang YS, Li XL, Li L, Tian BL. Preventing numerical oscillations in the flux-split based finite difference method for compressible flows with discontinuities. Journal of Computational Physics 2015. DOI: 10.1016/j.jcp.2015.07.049.

22. Steger JL, Warming RF. Flux vector splitting of the inviscid gasdynamic equations with applications to finite differerence methods. Journal of Computational Physics 1981; 40:263-293.

23. van Leer B. Flux vector splitting for Euler equation. ICASE Report No. 82-30, NASA Langley Research Center, Hampton, Virginia, 1982.

24. Harten A, Lax PD, van Leer B. On upstream difference and Godunov-type schemes for hyperbolic conservation laws. SIAM Review 1983; 25:35-61.

25. Shu CW, Osher S. Efficient implementation of essentially non-oscillatory shock-capturing schemes, II. Journal of Computational Physics 1989; 83:32-78.

26. Shu CW. Essentially non-oscillatory and weighted essentially non-oscillatory schemes for hyperbolic conservation laws. ICASE Report No. 97-65, NASA Langley Research Center, Hampton, Virginia, 1997.

27. Qiu JX, Shu CW. On the construction, comparison, and local characteristic decomposition for high-order central WENO schemes. Journal of Computational Physics 2002; 183:187-209.

28. Balsara DS, Shu CW. Monotonicity preserving weighted essentially nonoscillatory schemes with increasingly high order of accuracy. Journal of Computational Physics 2000; 160:405-452.

29. Henrick AK, Aslam TD, Powers JM. Mapped weighted essentially non-oscillatory schemes: achieving optimal order near critical points. Journal of Computational Physics 2005; 207:542-567.

30. Yamaleev NK, Carpenter MH. A systematic methodology for constructing high-order energy stable WENO schemes. Journal of Computational Physics 2009; 228:4248-4272.

31. Don WS, Borges R. Accuracy of the weighted essentially non-oscillatory conservative finite difference schemes. Journal of Computational Physics 2013; 250:347-372.

32. Jia FL, Gao Z, Don WS. A spectral study on the dissipation and dispersion of the WENO schemes. Journal of Scientific Computing 2014; 63:49-77.

33. Jiang GS, Shu CW. Efficient implementation of weighted ENO schemes. Journal of Computational Physics 1996; 126:202-228.

34. Gottlieb S, Shu CW. Total variation diminishing Runge-Kutta schemes. Mathematics of Computation 1998; 67: $73-85$. 